\title{
Baixa variedade na disponibilidade domiciliar de frutas e hortaliças no Brasil: dados das POF 2008-2009 e 2017-2018
}

\author{
Lack of variety of fruit and vegetables available in Brazilian \\ households: data from the Household Budget Surveys of 2008-2009 \\ and 2017-2018
}

Natália Oliveira (https://orcid.org/0000-0001-9852-7094) ${ }^{1}$
Fernanda Santin (https://orcid.org/0000-0002-8587-5273) ${ }^{2}$
Talita Rodrigues Paraizo (https://orcid.org/0000-0002-1845-1067) ${ }^{3}$
Jéssica Pereira Sampaio (https://orcid.org/0000-0003-3504-7776)
Nathália Moura-Nunes (https://orcid.org/0000-0002-7904-4844)
Daniela Silva Canella (https://orcid.org/0000-0001-9672-4983)

${ }^{1}$ Programa de Pós-

Graduação em Alimentação,

Nutrição e Saúde,

Universidade do Estado do

Rio de Janeiro (UERJ). R.

São Francisco Xavier 524,

Maracanã. 20550-013 Rio

de Janeiro RJ Brasil. nati.

olive@hotmail.com

${ }^{2}$ Núcleo de Pesquisas

Epidemiológicas em

Nutrição e Saúde,

Faculdade de Saúde Pública,

Universidade de São Paulo.

São Paulo SP Brasil.

${ }^{3}$ Residência em Nutrição,

UERJ. Rio de Janeiro RJ

Brasil.

${ }^{4}$ Instituto de Nutrição,

UERJ. Rio de Janeiro RJ

Brasil.

\begin{abstract}
The objective of this study was to establish the quantity and variety of fruit and vegetables (FV) available in Brazilian households in 2008-09 and 2017-18, and according to regions and income classes in 2017-18. Data from the Household Budget Surveys were used regarding household availability of fruit and vegetables. The annual per capita amount of each food item purchased was transformed into daily amounts. The absolute (gram/per capita/day) and the relative average quantities of each type of FV in Brazil (in both periods), and according to region and income (in 2017-18), were analyzed. An insufficient quantity of fruit and vegetables purchased in Brazil (54.4 $\mathrm{g}$ and $42.7 \mathrm{~g}$ in 2008-09; $49.7 \mathrm{~g}$ and $37.4 \mathrm{~g}$ in 2017-18, respectively) in all regions and income classes was verified. The South represented the highest amount purchased, while the North revealed the lowest; the quantity of FV increased as income increased. Six varieties of fruit (banana, apple, plantain, orange, watermelon, and papaya) and three types of vegetables (tomato, onion, and carrot) represented more than $50 \%$ of the total acquisition in Brazil, which was similar for all strata analyzed. The acquisition of fruit and vegetables in Brazil was low and featured little variation. This scenario was the same for all regions and income brackets.
\end{abstract}

Key words Diet surveys, Socioeconomic factors, Demography
Resumo O objetivo deste estudo foi caracterizar a quantidade e a variedade de frutas e hortaliças adquiridas nos domicílios brasileiros em 2008-09 e 2017-18 e segundo regiões e classes de rendimento em 2017-18. Foram utilizados dados das Pesquisas de Orçamentos Familiares referentes a disponibilidade domiciliar de frutas e hortaliças. A quantidade anual per capita adquirida de cada item alimentar foi transformada em valores diários. Foram descritas as médias da quantidade absoluta (grama/per capita/dia) e relativa de cada tipo de fruta e hortaliça para o Brasil (nos dois períodos) e segundo regiões e renda (em 2017-18). Verificou-se baixa aquisição de frutas e hortaliças para o Brasil (54,4 g e 42,7 $\mathrm{g}$ em 2008-09; $49,7 \mathrm{~g}$ e 37,4 g em 2017-18, respectivamente), e em todas as regiões e classes de renda analisadas. O Sul apresentou a maior aquisição e o Norte a menor; a quantidade adquirida aumentou com o aumento da renda. Seis tipos de frutas (banana prata, maçã, banana d'água, laranja pera, melancia e mamão) e três de hortaliças (tomate, cebola e cenoura) representaram mais de $50 \%$ da aquisição total no Brasil, sendo semelhante em todos os estratos analisados. A aquisição de frutas e hortaliças no Brasil foi baixa e apresentou pouca variação. Esse cenário se reproduziu em todas as regiões e faixas de renda.

Palavras-chave Inquéritos sobre dietas, Fatores socioeconômicos, Demografia 


\section{Introdução}

Frutas e hortaliças são alimentos que apresentam em sua composição nutrientes importantes, especialmente vitaminas e minerais, e outros componentes, como fibras e compostos fenólicos, que exercem efeitos positivos na saúde humana ${ }^{1,2}$. Devido à presença dessas substâncias, às propriedades biológicas que apresentam e às interações que estabelecem entre si nas diferentes matrizes alimentares, o consumo de frutas e hortaliças é considerado um importante fator de prevenção e proteção para o aparecimento e a progressão de doenças crônicas não transmissíveis (DCNT), como: doenças cardiovasculares, alguns tipos de câncer ${ }^{1,3}$, e para o excesso de adiposidade corporal e, consequentemente, a obesidade ${ }^{4}$.

O consumo adequado desses alimentos está associado a um menor risco de mortalidade por DCNT e por todas as causas ${ }^{3,5}$. O inverso também já foi evidenciado na literatura, visto que o consumo insuficiente de frutas e hortaliças foi apontado como a causa de cerca de 2,7 milhões de mortes por ano, em todo o mundo ${ }^{1}$, e de $19,3 \%$ das mortes por doença cardiovascular no Brasil em 2008-20096. Vale ressaltar que 4,4\% dessas mortes na população brasileira não foram prevenidas ou adiadas pelo consumo inadequado de frutas e hortaliças no período entre 1987-1988 e 2008-2009 ${ }^{6}$.

Com o intuito de reduzir a ocorrência de DCNT e controlar ou prevenir deficiências de micronutrientes, a Organização Mundial de Saúde (OMS) recomenda o consumo de 400 gramas de frutas e hortaliças por dia ${ }^{1}$. Ademais, o estímulo ao consumo de frutas e hortaliças ganhou destaque no Plano de Ações Estratégicas para o Enfrentamento das Doenças Crônicas não Transmissíveis no Brasil 2011-2022² e no Plano Nacional de Segurança Alimentar e Nutricional (PLANSAN 2016-2019) ${ }^{7}$, os quais têm como uma de suas metas o aumento do consumo destes alimentos. Ainda, o Ministério da Saúde destaca no Guia Alimentar para a População Brasileira a importância do consumo variado de frutas e hortaliças para aumentar e diversificar o consumo de nutrientes e outros compostos com atividade biológica benéfica para a saúde ${ }^{8}$. Reconhecendo a importância do componente variedade na qualidade da alimentação, o Programa Nacional de Alimentação Escolar (PNAE) incorporou em seus parâmetros (Resolução no 6 , de 20 de maio de 2020) recomendações específicas sobre número de alimentos a serem adquiridos, com vistas a promover maior variedade e diversidade na alimentação dos escolares ${ }^{9}$.
Quantitativamente, estima-se que o consumo de frutas e hortaliças no Brasil corresponda a menos da metade das recomendações nutricionais $^{10,11}$, apesar da grande variedade desses alimentos disponível no país ${ }^{12}$. Dados do Sistema de Vigilância de Fatores de Risco e Proteção para Doenças Crônicas por Inquérito Telefônico (Vigitel) apontam que, em 2019, apenas 22,9\% dos brasileiros consumiram a quantidade de frutas e hortaliças recomendada pela $\mathrm{OMS}^{13}$. De acordo com estudos anteriores, fatores socioeconômicos, como nível educacional e renda familiar, e demográficos são importantes determinantes do consumo de frutas e hortaliças ${ }^{14-16}$. Além disso, o consumo desses alimentos pode variar consideravelmente entre as regiões do país ${ }^{10}$.

Embora sejam numerosos os estudos sobre diversos aspectos relacionados ao consumo de frutas e hortaliças no Brasil, a disponibilidade domiciliar com enfoque na variedade destes alimentos tem sido pouco explorada. Sendo assim, o presente estudo teve como objetivo caracterizar a quantidade e a variedade de frutas e hortaliças disponíveis nos domicílios brasileiros em 20082009 e em 2017-2018 e segundo as cinco regiões do Brasil e diferentes classes de rendimento em 2017-2018.

\section{Métodos}

Foram utilizados dados das Pesquisas de Orçamentos Familiares (POF) realizadas pelo Instituto Brasileiro de Geografia e Estatística (IBGE) entre maio de 2008 e maio de $2009^{17}$ e entre julho de 2017 e julho de $2018^{18}$. Trata-se de inquérito com dados representativos da população brasileira, distribuída entre as cinco regiões do país, as áreas urbana e rural e em todas as faixas de renda.

As POF de 2008-2009 e 2017-2018 utilizaram plano amostral complexo, por conglomerados, envolvendo estratificação geográfica e socioeconômica de todos os setores censitários do país, seguida de sorteio aleatório de setores no primeiro estágio e de domićlios no segundo estágio. As amostras foram compostas por 55.970 domicílios, distribuídos em 550 estratos amostrais, para a POF 2008-2009; e 57.920 domicílios, distribuídos em 575 estratos amostrais, para a POF 2017-2018. As coletas de dados foram realizadas ao longo de 12 meses, de maneira uniforme nos estratos, garantindo a distribuição de domicílios nos quatro trimestres dos anos avaliados ${ }^{17,18}$.

No presente estudo, foram utilizados dados referentes a: disponibilidade domiciliar dos diferentes tipos de frutas e hortaliças registrados por 
todos os domicílios amostrados, considerando as regiões do país em que são localizados os domicílios e as classes de rendimento. O levantamento dos dados de 2008-2009 foi realizado a partir do Sistema IBGE de Recuperação Automática (SIDRA), que armazena os dados de forma agregada das pesquisas realizadas pelo IBGE. Para 2017-2018, foram utilizados os dados disponibilizados como "tabelas completas" no próprio site do IBGE, na seção POF 2017-2018 - Avaliação Nutricional da Disponibilidade Domiciliar de Alimentos no Brasil.

Para os dados referentes à disponibilidade de alimentos, em ambos os períodos, foi realizado o registro de todos os alimentos adquiridos para consumo domiciliar, anotados em caderneta específica pelos próprios moradores dos domicílios (ou entrevistador, quando necessário) durante sete dias consecutivos ${ }^{17,18}$. Devido ao curto período de referência empregado para o registro das despesas com alimentação em cada domicílio, foram utilizados como unidade de estudo os agregados de domicílios, definidos com base nos estratos amostrais, cujo padrão de aquisição anual de alimentos pode ser conhecido com maior precisão. Para tal, considerou-se o maior nível de desagregação de alimentos disponível.

A quantidade per capita de cada item alimentar adquirida em cada ano e expressa em quilogramas foi transformada em quantidade adquirida por dia e expressa em gramas, a fim de representar valores diários de consumo (grama/ per capita/dia). Posteriormente, foram aplicados fatores de correção a esses dados, com o intuito de excluir a fração não comestível, como cascas, e expressar as quantidades efetivamente disponíveis para consumo ${ }^{19}$.

Para avaliar a distribuição regional da aquisição de alimentos na POF 2017-2018, foram consideradas as cinco regiões brasileiras (Norte, Nordeste, Centro-Oeste, Sudeste e Sul). As classes de rendimento foram estimadas a partir de valores de rendimento total e variação patrimonial médio mensal familiar, que corresponde à soma dos rendimentos monetários mensais brutos, dos rendimentos não monetários mensais das unidades de consumo e da variação patrimonial, dividida pelo número de unidades de consumo. Para tanto, foram estabelecidas na POF 2017-2018 as seguintes classes de rendimento: até R\$ 1.908 (até 2 salários mínimos), mais de R $\$ 1.908$ a R $\$ 2.862$ (de 2 a 3 salários mínimos), mais de R\$ 2.862 a R\$ 5.724 (de 3 a 6 salários mínimos), mais de R\$ 5.724 a R\$ 9.540 (de 6 a 10 salários mínimos), mais de R\$ 9.540 a R 14.310 (de 10 a 15 salários mínimos), mais de R 14.310 (mais de 15 salários mínimos) ${ }^{18}$.

Para analisar a disponibilidade de frutas e hortaliças, foram considerados os valores médios das quantidades absoluta (gramas/per capita/ dia) e relativa (participação na quantidade total) de cada tipo de fruta e de hortaliça adquirido pelos domicílios brasileiros nos dois períodos investigados. Em seguida, considerando somente os dados da POF 2017-2018, foram analisadas as quantidades absoluta e relativa, estratificadas pelas cinco regiões brasileiras e pelas seis classes de rendimento. As estimativas levaram em conta os fatores de expansão da POF e a complexidade do desenho amostral da pesquisa.

\section{Resultados}

A disponibilidade domiciliar média de frutas e hortaliças no Brasil foi estimada em 97,1 gramas/ per capita/dia, sendo $54,4 \mathrm{~g}$ de frutas e $42,7 \mathrm{~g}$ de hortaliças, no período de 2008-2009, e em 87,1 $\mathrm{g}$, sendo $49,7 \mathrm{~g}$ de frutas e $37,4 \mathrm{~g}$ de hortaliças em 2017-2018. Em 2008-2009 as frutas mais adquiridas foram: melancia, banana prata, maçã, laranja pera, banana d'água e mamão, representando 53,4\% da quantidade total. As hortaliças mais adquiridas foram tomate, cebola e cenoura, que representam $56,0 \%$ da quantidade total. Já no período de 2017-2018, as frutas mais adquiridas foram: banana prata, maçã, banana d'água, laranja pera, melancia e mamão, representando $56,0 \%$ da quantidade total. Para hortaliças, o cenário se manteve o mesmo, com tomate, cebola e cenoura representando $58,0 \%$ da quantidade total (Tabela 1).

A quantidade adquirida de frutas em todas as regiões do Brasil em 2017-2018 foi baixa e foram observadas algumas diferenças em relação à distribuição da aquisição entre as regiões. A maior disponibilidade de frutas foi encontrada na região Sul $(60,8 \mathrm{~g})$ enquanto a menor foi observada na região Norte $(25,6 \mathrm{~g})$. Ressalta-se a participação expressiva de banana, melancia, laranja e maçã em todas as regiões. As seis frutas mais adquiridas de cada região, representaram entre 53,4\% (Centro-Oeste) e 58,5\% (Sudeste) do total adquirido. As frutas com maior aquisição em cada região foram: banana prata nas regiões Nordeste e Norte, laranja pera no Sudeste, banana d'água no Sul e melancia no Centro-Oeste. O mamão foi bastante adquirido em todas as regiões, exceto no Norte. Entre as bananas, a banana-d'água foi mais adquirida nas regiões Sul 
e Sudeste, enquanto a banana-prata teve um consumo maior nas regiões Norte e Nordeste (Tabela 2).

A aquisição de hortaliças em 2017-2018 também foi baixa em todas as regiões. A região Sul apresentou a maior quantidade adquirida $(44,2$ g), seguida pela região Centro-Oeste $(42,2 \mathrm{~g})$. Os menores valores foram encontrados para as regiões Norte e Nordeste $(20,2$ g e 33,7 g, respectivamente). Tomate, cebola e cenoura foram as hortaliças mais adquiridas em todas as regiões, representando mais de $50 \%$ da aquisição total em todas as regiões, variando de $53,3 \%$ (Sudeste) a 67,4\% (Nordeste) (Tabela 3).

Com relação a distribuição segundo classes de rendimento, observou-se que a aquisição tanto de frutas quanto de hortaliças aumentou conforme o aumento da renda (Tabela 4 e 5). A menor aquisição foi verificada na classe cujo rendimento era de até $\mathrm{R} \$ 1.908$, totalizando 26,9

Tabela 1. Disponibilidade absoluta e relativa (quantidade em gramas/per capita/dia e participação na quantidade total) de frutas e hortaliças em domicílios brasileiros. Brasil, 2008-2009 e 2017-2018.

\begin{tabular}{|c|c|c|c|c|c|}
\hline \multicolumn{3}{|c|}{ Frutas } & \multicolumn{3}{|c|}{ Hortaliças } \\
\hline \multirow[b]{2}{*}{ Tipos } & 2008-2009 & $2017-2018$ & \multirow[b]{2}{*}{ Tipos } & 2008-2009 & $2017-2018$ \\
\hline & $\begin{array}{c}\text { Quantidade } \\
(\%)\end{array}$ & $\begin{array}{c}\text { Quantidade } \\
(\%)\end{array}$ & & $\begin{array}{c}\text { Quantidade } \\
(\%)\end{array}$ & $\begin{array}{c}\text { Quantidade } \\
(\%)\end{array}$ \\
\hline Abacate & $0,5(1,0)$ & $0,6(1,2)$ & Acelga & $0,1(0,2)$ & $0,1(0,3)$ \\
\hline Abacaxi & $2,6(4,8)$ & $2,5(5,0)$ & Agrião & $0,1(0,2)$ & $0,1(0,2)$ \\
\hline Acerola & $0,3(0,6)$ & $0,3(0,5)$ & Alface & $2,1(4,8)$ & $1,6(4,2)$ \\
\hline Banana-d'água & $4,2(7,7)$ & $4,6(9,3)$ & Cheiro-verde & $0,5(1,2)$ & $0,5(1,3)$ \\
\hline Banana-da-terra & $0,8(1,5)$ & $0,9(1,8)$ & Couve & $0,6(1,5)$ & $0,6(1,6)$ \\
\hline Banana-maçã & $0,6(1,1)$ & $0,5(1,1)$ & Couve-brócolis & $0,4(0,9)$ & $0,3(0,9)$ \\
\hline Banana-prata & $5,3(9,8)$ & $5,8(11,8)$ & Couve-flor & $0,4(0,8)$ & $0,2(0,7)$ \\
\hline Outras bananas & $3,7(6,7)$ & $1,6(3,1)$ & Repolho & $2,4(5,5)$ & $1,6(4,3)$ \\
\hline Goiaba & $0,9(1,6)$ & $0,9(1,8)$ & $\begin{array}{l}\text { Outras hortaliças } \\
\text { folhosas }\end{array}$ & $0,7(1,7)$ & $0,6(1,6)$ \\
\hline Laranja-baía & $0,3(0,6)$ & $0,2(0,4)$ & Abóbora & $2,2(5,2)$ & $1,9(5,1)$ \\
\hline Laranja-lima & $0,7(1,2)$ & $1,1(2,3)$ & Abobrinha & $0,8(1,9)$ & $0,8(2,0)$ \\
\hline Laranja-pera & $5,0(9,1)$ & $4,5(9,0)$ & Berinjela & $0,4(0,9)$ & $0,4(1,0)$ \\
\hline Laranja-seleta & $0,2(0,3)$ & $0,2(0,4)$ & Cebola & $8,0(18,7)$ & $7,7(20,5)$ \\
\hline Outras laranjas & $3,5(6,4)$ & $1,5(3,1)$ & Chuchu & $1,6(3,7)$ & $1,3(3,5)$ \\
\hline Limão comum & $0,7(1,4)$ & $1,0(2,0)$ & Jiló & $0,3(0,8)$ & $0,3(0,8)$ \\
\hline Mamão & $4,0(7,3)$ & $3,5(7,0)$ & Maxixe & $0,1(0,2)$ & $0,1(0,2)$ \\
\hline Manga & $1,6(3,0)$ & $2,0(4,0)$ & Pepino fresco & $1,0(2,3)$ & $0,8(2,3)$ \\
\hline Maracujá & $0,5(0,9)$ & $0,6(1,2)$ & Pimentão & $1,3(3,1)$ & $1,1(2,8)$ \\
\hline Melancia & $5,4(10,0)$ & $4,3(8,6)$ & Quiabo & $0,6(1,4)$ & $0,5(1,3)$ \\
\hline Melão & $0,9(1,6)$ & $1,6(3,2)$ & Tomate & $12,3(28,8)$ & $10,5(28,1)$ \\
\hline Tangerina & $2,3(4,2)$ & $1,9(3,9)$ & Vagem & $0,4(1,0)$ & $0,2(0,6)$ \\
\hline $\begin{array}{l}\text { Outras frutas de clima } \\
\text { tropical }\end{array}$ & $0,8(1,5)$ & $0,6(1,3)$ & $\begin{array}{l}\text { Outras hortaliças } \\
\text { frutosas }\end{array}$ & $0,2(0,5)$ & $0,3(0,8)$ \\
\hline Ameixa & $0,3(0,6)$ & $0,3(0,5)$ & Alho & $1,1(2,7)$ & $1,2(3,2)$ \\
\hline Caqui & $0,4(0,8)$ & $0,4(0,8)$ & Beterraba & $1,1(2,6)$ & $0,9(2,5)$ \\
\hline Maçã & $5,2(9,5)$ & $5,1(10,3)$ & Cenoura & $3,7(8,6)$ & $3,5(9,4)$ \\
\hline Morango & $0,4(0,7)$ & $0,3(0,7)$ & $\begin{array}{l}\text { Outras hortaliças } \\
\text { tuberosas }\end{array}$ & $0,3(0,8)$ & $0,3(0,8)$ \\
\hline Pêra & $0,9(1,6)$ & $0,8(1,5)$ & & & \\
\hline Pêssego & $0,5(1,0)$ & $0,4(0,7)$ & & & \\
\hline Uva & $1,7(3,1)$ & $1,5(3,0)$ & & & \\
\hline $\begin{array}{l}\text { Outras frutas de clima } \\
\text { temperado }\end{array}$ & $0,2(0,4)$ & $0,2(0,5)$ & & & \\
\hline Total & $54,4(100,0)$ & $49,7(100,0)$ & Total & $42,7(100,0)$ & $37,4(100,0)$ \\
\hline
\end{tabular}

Fonte: Elaborado pelas autoras. 
g para frutas e 24,5 g para hortaliças. A maior quantidade adquirida foi observada na classe com rendimento maior que $\mathrm{R} \$ 14.310$, sendo a aquisição de frutas equivalente a $111,8 \mathrm{~g}$ e a de hortaliças 73,0 g. Em todas as classes de rendimentos, cerca de $50 \%$ da aquisição total de frutas e hortaliças foi representada por seis tipos de frutas (variando de $57,3 \%$ para as famílias mais pobres a $53,9 \%$ para as famílias mais ricas) e por três tipos de hortaliças (variando de 54,4\% para as famílias mais pobres a $63,3 \%$ para as famílias mais ricas). Esse percentual diminuiu gradativamente com o aumento da renda, indicando que os domicílios com menor renda, além de terem menor quantidade adquirida, também apresentavam uma menor variedade na compra destes alimentos (Tabela 4 e 5).

Tabela 2. Disponibilidade absoluta e relativa (quantidade em gramas/per capita/dia e participação na quantidade total) de frutas em domicílios brasileiros segundo regiões. Brasil, 2017-2018.

\begin{tabular}{|c|c|c|c|c|c|}
\hline \multirow{3}{*}{ Frutas } & \multicolumn{5}{|c|}{ Regiões } \\
\hline & Norte & Nordeste & Sudeste & Sul & $\begin{array}{c}\text { Centro- } \\
\text { Oeste } \\
\end{array}$ \\
\hline & $\begin{array}{c}\text { Quantidade } \\
(\%)\end{array}$ & $\begin{array}{c}\text { Quantidade } \\
(\%)\end{array}$ & $\begin{array}{c}\text { Quantidade } \\
(\%)\end{array}$ & $\begin{array}{c}\text { Quantidade } \\
(\%)\end{array}$ & $\begin{array}{c}\text { Quantidade } \\
(\%)\end{array}$ \\
\hline Abacate & $0,5(2,0)$ & $0,5(1,0)$ & $0,6(1,1)$ & $0,8(1,2)$ & $0,5(1,1)$ \\
\hline Abacaxi & $1,5(5,8)$ & $3,1(6,9)$ & $2,3(4,3)$ & $2,3(3,8)$ & $2,5(5,0)$ \\
\hline Acerola & $0,4(1,7)$ & $0,6(1,4)$ & $0,1(0,2)$ & $0,0(0,0)$ & $0,1(0,3)$ \\
\hline Banana-d'água & $1,1(4,3)$ & $0,9(2,0)$ & $6,2(11,6)$ & $9,4(15,5)$ & $4,2(8,2)$ \\
\hline Banana-da-terra & $1,1(4,3)$ & $2,0(4,4)$ & $0,5(0,9)$ & $0,1(0,2)$ & $0,6(1,2)$ \\
\hline Banana-maçã & $0,5(1,9)$ & $0,3(0,7)$ & $0,5(0,9)$ & $0,3(0,4)$ & $2,1(4,1)$ \\
\hline Banana-prata & $3,9(15,4)$ & $8,1(18,1)$ & $5,5(10,2)$ & $4,7(7,8)$ & $4,1(8,1)$ \\
\hline Outras bananas & $1,2(4,7)$ & $2,1(4,7)$ & $1,3(2,4)$ & $1,2(2,0)$ & $2,1(4,1)$ \\
\hline Goiaba & $0,5(1,8)$ & $1,7(3,9)$ & $0,6(1,1)$ & $0,5(0,9)$ & $0,5(1,1)$ \\
\hline Laranja-baía & $0,0(0,0)$ & $0,1(0,2)$ & $0,1(0,2)$ & $1,0(1,6)$ & $0,1(0,1)$ \\
\hline Laranja-lima & $0,5(1,9)$ & $0,9(1,9)$ & $1,5(2,7)$ & $1,1(1,8)$ & $1,1(2,2)$ \\
\hline Laranja-pera & $1,5(6,0)$ & $3,0(6,6)$ & $6,3(11,7)$ & $3,9(6,4)$ & $4,3(8,5)$ \\
\hline Laranja-seleta & $0,3(1,2)$ & $0,1(0,2)$ & $0,3(0,6)$ & $0,2(0,3)$ & $0,2(0,5)$ \\
\hline Outras laranjas & $1,3(5,0)$ & $1,6(3,5)$ & $1,4(2,6)$ & $1,5(2,5)$ & $2,6(5,1)$ \\
\hline Limão comum & $0,7(2,8)$ & $0,5(1,1)$ & $1,4(2,6)$ & $1,0(1,7)$ & $1,0(2,0)$ \\
\hline Mamão & $1,1(4,2)$ & $2,7(6,0)$ & $4,3(7,9)$ & $4,4(7,2)$ & $3,2(6,3)$ \\
\hline Manga & $0,5(1,9)$ & $1,7(3,8)$ & $2,4(4,4)$ & $2,5(4,1)$ & $1,3(2,7)$ \\
\hline Maracujá & $0,3(1,1)$ & $1,0(2,2)$ & $0,5(1,0)$ & $0,2(0,4)$ & $0,6(1,2)$ \\
\hline Melancia & $3,3(13,0)$ & $4,2(9,4)$ & $3,6(6,6)$ & $5,9(9,7)$ & $6,2(12,3)$ \\
\hline Melão & $0,9(3,5)$ & $1,7(3,7)$ & $1,6(2,9)$ & $1,8(3,0)$ & $1,9(3,7)$ \\
\hline Tangerina & $0,4(1,5)$ & $0,8(1,7)$ & $2,4(4,4)$ & $3,8(6,3)$ & $1,8(3,6)$ \\
\hline Outras frutas de clima tropical & $0,6(2,3)$ & $0,8(1,7)$ & $0,5(1,0)$ & $0,6(0,9)$ & $1,0(2,0)$ \\
\hline Ameixa & $0,0(0,2)$ & $0,1(0,2)$ & $0,3(0,6)$ & $0,5(0,8)$ & $0,3(0,6)$ \\
\hline Caqui & $0,1(0,2)$ & $0,0(0,1)$ & $0,4(0,8)$ & $1,2(2,0)$ & $0,4(0,7)$ \\
\hline Maçã & $2,4(9,5)$ & $4,2(9,3)$ & $5,7(10,5)$ & $6,9(11,4)$ & $5,0(10,0)$ \\
\hline Morango & $0,1(0,4)$ & $0,1(0,3)$ & $0,4(0,8)$ & $0,7(1,1)$ & $0,3(0,6)$ \\
\hline Pêra & $0,3(1,0)$ & $0,4(0,9)$ & $1,1(2,1)$ & $0,6(1,0)$ & $0,8(1,6)$ \\
\hline Pêssego & $0,0(0,0)$ & $0,0(0,1)$ & $0,4(0,8)$ & $1,2(1,9)$ & $0,2(0,3)$ \\
\hline Uva & $0,6(2,3)$ & $1,7(3,8)$ & $1,4(2,5)$ & $2,1(3,4)$ & $1,2(2,4)$ \\
\hline Outras frutas de clima temperado & $0,0(0,1)$ & $0,1(0,2)$ & $0,3(0,6)$ & $0,4(0,7)$ & $0,2(0,4)$ \\
\hline Total & $25,6(100,0)$ & $45,0(100,0)$ & $53,9(100,0)$ & $60,8(100,0)$ & $50,4(100,0)$ \\
\hline
\end{tabular}

Fonte: Elaborado pelas autoras. 
Tabela 3. Disponibilidade absoluta e relativa (quantidade em gramas/per capita/dia e participação na quantidade total) de hortaliças em domicílios brasileiros segundo regiões. Brasil, 2017-2018.

\begin{tabular}{|c|c|c|c|c|c|}
\hline \multirow{3}{*}{ Hortaliças } & \multicolumn{5}{|c|}{ Regiões } \\
\hline & Norte & Nordeste & Sudeste & Sul & $\begin{array}{c}\text { Centro- } \\
\text { Oeste }\end{array}$ \\
\hline & $\begin{array}{c}\text { Quantidade } \\
(\%)\end{array}$ & $\begin{array}{c}\text { Quantidade } \\
(\%)\end{array}$ & $\begin{array}{c}\text { Quantidade } \\
(\%)\end{array}$ & $\begin{array}{c}\text { Quantidade } \\
(\%)\end{array}$ & $\begin{array}{c}\text { Quantidade } \\
(\%)\end{array}$ \\
\hline Acelga & $0,0(0,2)$ & $0,0(0,1)$ & $0,1(0,4)$ & $0,1(0,2)$ & $0,1(0,1)$ \\
\hline Agrião & $0,0(0,0)$ & $0,0(0,0)$ & $0,1(0,4)$ & $0,1(0,2)$ & $0,1(0,1)$ \\
\hline Alface & $0,4(2,2)$ & $0,7(2,2)$ & $1,8(4,6)$ & $2,7(6,2)$ & $1,9(4,5)$ \\
\hline Cheiro-verde & $0,8(4,0)$ & $0,7(2,0)$ & $0,3(0,8)$ & $0,6(1,3)$ & $0,4(0,9)$ \\
\hline Couve & $0,4(2,0)$ & $0,3(0,8)$ & $0,8(2,0)$ & $0,7(1,5)$ & $0,5(1,2)$ \\
\hline Couve-brócolis & $0,1(0,6)$ & $0,1(0,2)$ & $0,5(1,2)$ & $0,6(1,3)$ & $0,2(0,5)$ \\
\hline Couve-flor & $0,0(0,2)$ & $0,0(0,1)$ & $0,4(0,9)$ & $0,4(0,9)$ & $0,2(0,5)$ \\
\hline Repolho & $0,9(4,2)$ & $0,8(2,5)$ & $1,6(4,0)$ & $3,4(7,6)$ & $2,1(5,0)$ \\
\hline Outras hortaliças folhosas & $0,3(1,6)$ & $0,5(1,5)$ & $0,7(1,8)$ & $0,7(1,5)$ & $0,3(0,8)$ \\
\hline Abóbora & $0,8(3,8)$ & $1,7(5,0)$ & $2,2(5,4)$ & $2,0(4,5)$ & $2,2(5,2)$ \\
\hline Abobrinha & $0,1(0,6)$ & $0,2(0,6)$ & $1,1(2,8)$ & $0,7(1,7)$ & $1,3(3,2)$ \\
\hline Berinjela & $0,1(0,5)$ & $0,1(0,4)$ & $0,7(1,6)$ & $0,3(0,7)$ & $0,2(0,5)$ \\
\hline Cebola & $5,7(28,0)$ & $8,7(26,0)$ & $7,0(17,3)$ & $8,9(20,3)$ & $7,5(17,6)$ \\
\hline Chuchu & $0,2(1,0)$ & $1,3(3,7)$ & $1,5(3,8)$ & $1,4(3,2)$ & $1,1(2,7)$ \\
\hline Jiló & $0,1(0,4)$ & $0,1(0,2)$ & $0,5(1,3)$ & $0,1(0,1)$ & $0,9(2,1)$ \\
\hline Maxixe & $0,2(0,8)$ & $0,2(0,5)$ & $0,1(0,4)$ & $0,0(0,0)$ & $0,1(0,1)$ \\
\hline Pepino fresco & $0,7(3,4)$ & $0,5(1,5)$ & $0,8(2,1)$ & $1,6(3,5)$ & $1,0(2,3)$ \\
\hline Pimentão & $0,6(3,0)$ & $1,6(4,8)$ & $1,0(2,5)$ & $0,7(1,7)$ & $0,6(1,5)$ \\
\hline Quiabo & $0,1(0,6)$ & $0,4(1,1)$ & $0,8(1,9)$ & $0,1(0,2)$ & $0,7(1,6)$ \\
\hline Tomate & $5,3(26,3)$ & $10,5(31,1)$ & $10,6(26,4)$ & $11,7(26,5)$ & $13,9(32,8)$ \\
\hline Vagem & $0,0(0,2)$ & $0,0(0,1)$ & $0,3(0,9)$ & $0,4(1,0)$ & $0,2(0,6)$ \\
\hline Outras hortaliças frutosas & $0,4(1,7)$ & $0,2(0,5)$ & $0,2(0,5)$ & $0,7(1,7)$ & $0,2(0,6)$ \\
\hline Alho & $0,8(3,8)$ & $0,9(2,5)$ & $1,6(4,0)$ & $0,7(1,7)$ & $1,5(3,5)$ \\
\hline Beterraba & $0,4(1,9)$ & $0,6(1,9)$ & $1,0(2,5)$ & $1,5(3,3)$ & $1,0(2,5)$ \\
\hline Cenoura & $1,7(8,3)$ & $3,5(10,3)$ & $3,9(9,6)$ & $3,6(8,1)$ & $3,7(8,8)$ \\
\hline Outras hortaliças tuberosas & $0,1(0,7)$ & $0,1(0,4)$ & $0,4(0,9)$ & $0,5(1,1)$ & $0,3(0,8)$ \\
\hline Total & $20,2(100,0)$ & $33,7(100,0)$ & $40,0(100,0)$ & $44,2(100,0)$ & $42,2(100,0)$ \\
\hline
\end{tabular}

Fonte: Elaborado pelas autoras.

\section{Discussão}

A disponibilidade domiciliar de frutas e hortaliças no Brasil, nos períodos de 2008-2009 e 20172018, é considerada baixa quando comparada com a recomendação da OMS $(400 \mathrm{~g})^{1}$. Ainda, a despeito da inexistência de ponto de corte, podese considerar que a variedade de frutas e hortaliças adquiridas, considerando os tipos de alimentos, foi baixa no país, nas cinco regiões e em todas as classes de rendimentos.

O consumo insuficiente de frutas e hortaliças, bem como a baixa variedade destes alimentos, pode comprometer os efeitos de proteção e prevenção associados a seu consumo. A presença de nutrientes e de compostos bioativos, e a in- teração sinérgica desses componentes na matriz alimentar são os principais componentes responsáveis pelo atributo de proteção/prevenção para DCNT $^{20,21}$. A ausência ou presença em quantidades insuficientes de compostos fenólicos na alimentação pode inviabilizar as ações benéficas que exercem na saúde humana por meio de suas propriedades antioxidante, vasodilatadora, anti -inflamatória, anti-aterogênica e antitrombótica. O baixo consumo de frutas e hortaliças foi apontado, em outros estudos, como a principal razão pela baixa ingestão de compostos fenólicos ${ }^{21,22}$.

No período de 2017-2018, nenhuma das regiões brasileiras ou das classes de rendimento, de forma semelhante ao cenário nacional, apresentou aquisição de frutas e hortaliças em quanti- 
Tabela 4. Disponibilidade absoluta e relativa (quantidade em gramas/per capita/dia e participação na quantidade total) de frutas em domicílios brasileiros segundo classes de rendimento. Brasil, 2017-2018.

\begin{tabular}{|c|c|c|c|c|c|c|}
\hline \multirow{3}{*}{ Frutas } & \multicolumn{6}{|c|}{ Classes de rendimento } \\
\hline & até $\mathrm{R} \$ 1.908$ & $\begin{array}{c}\mathrm{R} \$ 1.908 \mathrm{a} \\
2.862\end{array}$ & $\begin{array}{c}\mathrm{R} \$ 2.862 \mathrm{a} \\
5.724\end{array}$ & $\begin{array}{l}\mathrm{R} \$ 5.724 \mathrm{a} \\
9.540\end{array}$ & $\begin{array}{c}\mathrm{R} \$ 9.540 \mathrm{a} \\
14.310\end{array}$ & $\begin{array}{c}\text { mais de R\$ } \\
14.310\end{array}$ \\
\hline & $\begin{array}{c}\text { Quantidade } \\
(\%)\end{array}$ & $\begin{array}{c}\text { Quantidade } \\
(\%)\end{array}$ & $\begin{array}{c}\text { Quantidade } \\
(\%)\end{array}$ & $\begin{array}{c}\text { Quantidade } \\
(\%)\end{array}$ & $\begin{array}{c}\text { Quantidade } \\
(\%)\end{array}$ & $\begin{array}{c}\text { Quantidade } \\
(\%)\end{array}$ \\
\hline Abacate & $0,2(0,9)$ & $0,3(0,9)$ & $0,5(1,1)$ & $0,8(1,3)$ & $1,1(1,2)$ & $1,8(1,6)$ \\
\hline Abacaxi & $1,2(4,4)$ & $1,7(4,6)$ & $2,6(5,6)$ & $2,8(4,6)$ & $4,6(5,1)$ & $5,3(4,8)$ \\
\hline Acerola & $0,2(0,8)$ & $0,3(0,9)$ & $0,2(0,5)$ & $0,3(0,5)$ & $0,2(0,3)$ & $0,3(0,3)$ \\
\hline Banana-d'água & $2,6(9,8)$ & $3,7(9,9)$ & $4,6(10,1)$ & $6,3(10,2)$ & $7,2(8,1)$ & $7,6(6,8)$ \\
\hline Banana-da-terra & $0,7(2,6)$ & $1,1(2,8)$ & $0,8(1,6)$ & $0,9(1,5)$ & $1,2(1,3)$ & $1,7(1,5)$ \\
\hline Banana-maçã & $0,3(1,1)$ & $0,5(1,3)$ & $0,4(0,9)$ & $0,7(1,1)$ & $0,7(0,8)$ & $1,3(1,2)$ \\
\hline Banana-prata & $3,9(14,4)$ & $5,5(14,7)$ & $5,3(11,4)$ & $6,5(10,6)$ & $9,5(10,6)$ & $11,4(10,2)$ \\
\hline Outras bananas & $1,3(4,9)$ & $1,6(4,2)$ & $1,5(3,2)$ & $1,4(2,3)$ & $2,0(2,2)$ & $2,5(2,2)$ \\
\hline Goiaba & $0,7(2,4)$ & $0,7(2,0)$ & $0,8(1,8)$ & $1,1(1,8)$ & $1,1(1,2)$ & $1,5(1,3)$ \\
\hline Laranja-baía & $0,1(0,2)$ & $0,1(0,4)$ & $0,2(0,5)$ & $0,3(0,5)$ & $0,6(0,6)$ & $0,4(0,3)$ \\
\hline Laranja-lima & $0,5(2,0)$ & $1,1(2,8)$ & $1,2(2,5)$ & $1,6(2,6)$ & $1,5(1,7)$ & $1,6(1,4)$ \\
\hline Laranja-pera & $2,0(7,6)$ & $2,8(7,4)$ & $4,1(8,9)$ & $5,4(8,8)$ & $9,2(10,3)$ & $12,6(11,3)$ \\
\hline Laranja-seleta & $0,2(0,9)$ & $0,1(0,4)$ & $0,2(0,5)$ & $0,2(0,3)$ & $0,4(0,4)$ & $0,4(0,3)$ \\
\hline Outras laranjas & $1,1(4,1)$ & $1,2(3,3)$ & $1,6(3,4)$ & $1,5(2,5)$ & $2,2(2,5)$ & $2,7(2,4)$ \\
\hline Limão comum & $0,3(1,3)$ & $0,6(1,5)$ & $1,0(2,1)$ & $1,3(2,2)$ & $2,1(2,3)$ & $2,9(2,6)$ \\
\hline Mamão & $1,3(4,7)$ & $1,9(5,1)$ & $2,7(5,8)$ & $4,7(7,7)$ & $8,5(9,5)$ & $11,6(10,4)$ \\
\hline Manga & $1,0(3,8)$ & $1,5(4,0)$ & $1,7(3,6)$ & $2,5(4,1)$ & $3,7(4,2)$ & $4,8(4,3)$ \\
\hline Maracujá & $0,4(1,4)$ & $0,4(1,2)$ & $0,6(1,4)$ & $0,6(1,1)$ & $0,9(1,0)$ & $1,1(1,0)$ \\
\hline Melancia & $2,4(8,9)$ & $3,6(9,6)$ & $4,0(8,7)$ & $5,1(8,3)$ & $8,5(9,5)$ & $7,4(6,6)$ \\
\hline Melão & $0,6(2,2)$ & $0,9(2,4)$ & $1,2(2,6)$ & $2,2(3,6)$ & $3,4(3,8)$ & $5,8(5,2)$ \\
\hline Tangerina & $0,8(2,9)$ & $1,1(3,0)$ & $2,0(4,2)$ & $2,6(4,2)$ & $3,4(3,8)$ & $4,9(4,4)$ \\
\hline Outras frutas de clima tropical & $0,5(2,0)$ & $0,6(1,6)$ & $0,6(1,3)$ & $0,5(0,9)$ & $0,8(0,9)$ & $1,5(1,4)$ \\
\hline Ameixa & $0,0(0,2)$ & $0,1(0,4)$ & $0,2(0,5)$ & $0,3(0,4)$ & $0,8(0,9)$ & $0,8(0,7)$ \\
\hline Caqui & $0,2(0,7)$ & $0,1(0,4)$ & $0,3(0,7)$ & $0,6(1,0)$ & $0,8(0,9)$ & $1,2(1,1)$ \\
\hline Maçã & $2,8(10,4)$ & $3,8(10,4)$ & $5,0(10,8)$ & $6,9(11,3)$ & $8,3(9,3)$ & $9,6(8,6)$ \\
\hline Morango & $0,1(0,2)$ & $0,1(0,4)$ & $0,3(0,7)$ & $0,5(0,8)$ & $0,8(0,9)$ & $1,2(1,0)$ \\
\hline Pêra & $0,2(0,8)$ & $0,4(1,1)$ & $0,7(1,5)$ & $1,0(1,7)$ & $1,7(1,9)$ & $2,3(2,1)$ \\
\hline Pêssego & $0,1(0,2)$ & $0,2(0,4)$ & $0,3(0,8)$ & $0,5(0,8)$ & $1,0(1,2)$ & $1,3(1,1)$ \\
\hline Uva & $1,1(4,0)$ & $1,0(2,6)$ & $1,3(2,8)$ & $1,7(2,8)$ & $2,5(2,8)$ & $3,5(3,2)$ \\
\hline $\begin{array}{l}\text { Outras frutas de clima } \\
\text { temperado }\end{array}$ & $0,1(0,2)$ & $0,1(0,3)$ & $0,2(0,5)$ & $0,3(0,5)$ & $0,7(0,8)$ & $0,8(0,7)$ \\
\hline Total & $26,9(100,0)$ & $37,1(100,0)$ & $46,1(100,0)$ & $61,1(100,0)$ & $89,4(100,0)$ & $111,8(100,0)$ \\
\hline
\end{tabular}

Fonte: Elaborado pelas autoras.

dade adequada ${ }^{1}$. Os resultados foram ainda mais preocupantes nas regiões Norte e Nordeste e entre os domicílios com rendimentos mais baixos, pois a quantidade adquirida foi diretamente relacionada com a renda.

Tanto em 2008-2009, quanto em 2017-2018, nove itens alimentares (tomate, cebola, banana prata, maçã, melancia, laranja pera, banana d'água, mamão e cenoura) representaram boa parte da alimentação dos brasileiros no que tange ao consumo de frutas e hortaliças, evidenciando pouca variedade na aquisição destes alimentos. Esta distribuição se comportou de maneira semelhante entre as regiões e as classes de rendimento na avaliação de 2017-2018.

Em consonância com a baixa aquisição de frutas e hortaliças observada no presente estudo, a literatura aponta que apenas $34,7 \%$ dos brasileiros consomem regularmente (cinco ou mais vezes por semana) frutas e hortaliças ${ }^{16}$. Com re- 
Tabela 5. Disponibilidade absoluta e relativa (quantidade em gramas/per capita/dia e participação na quantidade total) de hortaliças em domicílios brasileiros segundo classes de rendimento. Brasil, 2017-2018.

\begin{tabular}{|c|c|c|c|c|c|c|}
\hline \multirow{3}{*}{ Hortaliças } & \multicolumn{6}{|c|}{ Classes de rendimento } \\
\hline & até $\mathrm{R} \$ 1.908$ & $\begin{array}{c}\mathrm{R} \$ 1.908 \mathrm{a} \\
2.862 \\
\end{array}$ & $\begin{array}{c}\mathrm{R} \$ 2.862 \mathrm{a} \\
5.724 \\
\end{array}$ & $\begin{array}{c}\mathrm{R} \$ 5.724 \mathrm{a} \\
\quad 9.540 \\
\end{array}$ & $\begin{array}{c}\mathrm{R} \$ 9.540 \mathrm{a} \\
14.310 \\
\end{array}$ & $\begin{array}{c}\text { mais de R\$ } \\
14.310\end{array}$ \\
\hline & $\begin{array}{c}\text { Quantidade } \\
(\%)\end{array}$ & $\begin{array}{c}\text { Quantidade } \\
(\%)\end{array}$ & $\begin{array}{c}\text { Quantidade } \\
(\%)\end{array}$ & $\begin{array}{c}\text { Quantidade } \\
(\%)\end{array}$ & $\begin{array}{c}\text { Quantidade } \\
(\%)\end{array}$ & $\begin{array}{c}\text { Quantidade } \\
(\%)\end{array}$ \\
\hline Acelga & $0,0(0,1)$ & $0,0(0,1)$ & $0,1(0,2)$ & $0,1(0,3)$ & $0,3(0,5)$ & $0,4(0,5)$ \\
\hline Agrião & $0,0(0,1)$ & $0,0(0,1)$ & $0,1(0,2)$ & $0,1(0,3)$ & $0,2(0,3)$ & $0,2(0,3)$ \\
\hline Alface & $0,8(3,4)$ & $1,2(3,9)$ & $1,7(4,5)$ & $1,8(4,3)$ & $2,5(4,3)$ & $2,9(4,0)$ \\
\hline Cheiro-verde & $0,5(1,9)$ & $0,5(1,5)$ & $0,5(1,4)$ & $0,5(1,1)$ & $0,5(1,0)$ & $0,6(0,9)$ \\
\hline Couve & $0,3(1,4)$ & $0,5(1,5)$ & $0,6(1,7)$ & $0,7(1,6)$ & $0,8(1,4)$ & $1,1(1,5)$ \\
\hline Couve-brócolis & $0,1(0,3)$ & $0,1(0,4)$ & $0,3(0,7)$ & $0,5(1,1)$ & $0,8(1,4)$ & $1,4(1,9)$ \\
\hline Couve-flor & $0,1(0,2)$ & $0,1(0,4)$ & $0,2(0,6)$ & $0,3(0,8)$ & $0,7(1,2)$ & $0,9(1,2)$ \\
\hline Repolho & $0,9(3,6)$ & $1,1(3,6)$ & $1,8(4,9)$ & $2,0(4,8)$ & $2,7(4,8)$ & $2,5(3,5)$ \\
\hline $\begin{array}{l}\text { Outras hortaliças } \\
\text { folhosas }\end{array}$ & $0,3(1,4)$ & $0,5(1,5)$ & $0,5(1,4)$ & $0,6(1,4)$ & $0,8(1,4)$ & $1,9(2,6)$ \\
\hline Abóbora & $1,2(5,0)$ & $2,0(6,5)$ & $1,8(5,0)$ & $2,0(4,7)$ & $2,6(4,6)$ & $3,4(4,7)$ \\
\hline Abobrinha & $0,4(1,6)$ & $0,5(1,6)$ & $0,6(1,8)$ & $0,9(2,0)$ & $1,5(2,6)$ & $2,3(3,1)$ \\
\hline Berinjela & $0,1(0,4)$ & $0,2(0,6)$ & $0,3(0,7)$ & $0,4(1,0)$ & $0,7(1,3)$ & $2,0(2,7)$ \\
\hline Cebola & $6,0(24,3)$ & $6,4(20,9)$ & $7,4(20,2)$ & $8,6(20,2)$ & $10,5(18,4)$ & $13,4(18,3)$ \\
\hline Chuchu & $0,8(3,1)$ & $1,1(3,5)$ & $1,3(3,5)$ & $1,5(3,5)$ & $2,2(3,9)$ & $2,5(3,4)$ \\
\hline Jiló & $0,1(0,6)$ & $0,3(1,0)$ & $0,4(1,2)$ & $0,3(0,8)$ & $0,5(0,8)$ & $0,3(0,4)$ \\
\hline Maxixe & $0,2(0,7)$ & $0,1(0,5)$ & $0,1(0,3)$ & $0,0(0,1)$ & $0,1(0,2)$ & $0,1(0,1)$ \\
\hline Pepino fresco & $0,5(1,9)$ & $0,6(1,9)$ & $0,8(2,2)$ & $1,0(2,4)$ & $1,6(2,7)$ & $1,8(2,5)$ \\
\hline Pimentão & $0,8(3,1)$ & $0,9(3,0)$ & $1,0(2,7)$ & $1,2(2,7)$ & $1,8(3,1)$ & $1,8(2,5)$ \\
\hline Quiabo & $0,3(1,4)$ & $0,5(1,7)$ & $0,6(1,6)$ & $0,4(1,0)$ & $0,5(0,9)$ & $0,7(1,0)$ \\
\hline Tomate & $7,4(30,2)$ & $9,0(29,4)$ & $9,9(27,3)$ & $11,8(27,8)$ & $15,9(27,9)$ & $19,7(27,0)$ \\
\hline Vagem & $0,0(0,2)$ & $0,1(0,5)$ & $0,2(0,6)$ & $0,4(1,0)$ & $0,6(1,0)$ & $0,6(0,8)$ \\
\hline $\begin{array}{l}\text { Outras hortaliças } \\
\text { frutosas }\end{array}$ & $0,1(0,5)$ & $0,2(0,5)$ & $0,3(0,7)$ & $0,4(1,0)$ & $0,4(0,6)$ & $0,9(1,2)$ \\
\hline Alho & $0,8(3,4)$ & $1,0(3,2)$ & $1,2(3,4)$ & $1,4(3,3)$ & $1,6(2,8)$ & $1,9(2,6)$ \\
\hline Beterraba & $0,5(2,1)$ & $0,7(2,2)$ & $1,1(3,0)$ & $1,0(2,4)$ & $1,3(2,3)$ & $1,6(2,2)$ \\
\hline Cenoura & $2,2(8,8)$ & $3,0(9,7)$ & $3,5(9,7)$ & $4,1(9,6)$ & $5,1(8,9)$ & $6,6(9,1)$ \\
\hline $\begin{array}{l}\text { Outras hortaliças } \\
\text { tuberosas }\end{array}$ & $0,1(0,3)$ & $0,1(0,3)$ & $0,2(0,5)$ & $0,3(0,8)$ & $1,0(1,7)$ & $1,5(2,0)$ \\
\hline Total & $24,5(100,0)$ & $30,7(100,0)$ & $36,5(100,0)$ & $42,3(100,0)$ & $57,2(100,0)$ & $73,0(100,0)$ \\
\hline
\end{tabular}

lação às regiões, Norte e Nordeste apresentaram menor participação energética de frutas e hortaliças na alimentação ${ }^{10}$ e menor frequência de indivíduos com consumo regular destes alimen$\operatorname{tos}^{14,16}$. Considerando as classes de rendimentos, resultados semelhantes foram verificados em estudos utilizando dados das POF anteriores, evidenciando a relação direta da renda familiar com a aquisição e consumo de frutas e hortaliças ${ }^{15}$. Maior renda foi também associada com maior ingestão de compostos fenólicos ${ }^{21}$.

A inadequação da quantidade de frutas e hortaliças consumidas, no entanto, não é veri- ficada apenas no Brasil. Estudos realizados na Austrália $^{23}$, Nova Zelândia ${ }^{24}$, Alemanha ${ }^{25}$ e Coreia do $\mathrm{Sul}^{26}$ também revelaram grande parcela da população com consumo insuficiente desses alimentos. Em especial, o estudo realizado com a população australiana verificou que a maioria dos indivíduos não alcança as recomendações do país: uma em cada dez pessoas atinge o consumo diário recomendado de hortaliças e metade atinge o recomendado de frutas ${ }^{23}$.

De uma forma geral, a renda dos indivíduos ou famílias e o custo dos alimentos são dois importantes determinantes do baixo consumo 
de frutas e hortaliças ${ }^{15,23,27}$. Ainda, outras barreiras dificultam o alcance da recomendação para consumo desses alimentos, como a baixa oferta no comércio local, a dificuldade de transporte e a carência de políticas públicas com foco no aces$\mathrm{so}^{28}$. Essas características - que são inerentes ao ambiente alimentar - são apontadas como importantes influenciadores do consumo alimen$\operatorname{tar}^{29,30}$. Estudo realizado em São Paulo verificou correlação positiva entre o consumo regular de frutas e hortaliças e o nível socioeconômico da área de residência, por ser mais difícil a aquisição em regiões mais pobres ${ }^{31}$. Características individuais como a falta de tempo, de conhecimento sobre a variedade de alimentos que existem e/ou sobre a importância do seu consumo para a saúde e falta de hábito de consumo também são elementos apontados como dificultadores. Já outros fatores atuam como promotores do consumo desses alimentos: busca pela saúde e prevenção de doenças, desejo de emagrecer, preço baixo e boa qualidade dos alimentos, proximidade do comércio e hábitos alimentares ${ }^{28}$.

Além da quantidade, a variedade no consumo de frutas e hortaliças também é um fator importante na prevenção de doenças crônicas e adiposidade ${ }^{4}$. No entanto, os resultados encontrados no presente estudo apontam uma prática oposta a isto no Brasil. Apesar de não existir ponto de corte para a definição da baixa ou alta variedade, acreditamos que em um cenário no qual foram avaliados 49 alimentos e somente nove destes representaram mais de $50 \%$ da aquisição total, pode-se considerar que há baixa variedade, caracterizando monotonia alimentar.

A monotonia alimentar resulta na ingestão dos mesmos micronutrientes e compostos bioativos e/ou das mesmas quantidades desses compostos. A exposição restrita a poucos representantes dessas classes de substâncias ou o consumo desses compostos em quantidades insuficientes pode levar à redução dos seus benefícios à saú$\mathrm{de}^{20,21}$. O padrão pouco variado de aquisição já foi evidenciado também para as hortaliças em 20082009, uma vez que somente 10 tipos de hortaliças responderam por mais de $80 \%$ da participação destas na disponibilidade domiciliar no Brasil ${ }^{11}$. A baixa variedade de consumo também foi verificada nos Estados Unidos, sendo que tomate, alface e cebola responderam pela maior fração de hortaliças consumidas, enquanto maçã e banana foram as frutas in natura mais consumidas ${ }^{32}$.

O Brasil é um país que apresenta grande diversidade de alimentos disponíveis, com variação nos hábitos alimentares devido a fatores econô- micos, sociais, étnicos, culturais e regionais ${ }^{8,12}$. Frutas como laranja e banana, são bastante consumidas em todas as regiões. $\mathrm{O}$ abacate apresenta um consumo elevado na região Norte, onde é utilizado em diversas preparações regionais, salgadas e doces, no entanto, não é muito consumido nas demais regiões. O mesmo é percebido com relação às hortaliças. Alface e tomate são bastante consumidos em todas as regiões (com destaque para o Sul e Sudeste), porém o maxixe, que é um alimento característico do Norte e do Nordeste, apresenta maior consumo nessas regiões ${ }^{12}$. Apesar da variedade de frutas e hortaliças disponíveis no Brasil, em nosso estudo verificamos relativa monotonia na aquisição desses alimentos em todas as regiões e as classes de rendimentos, com destaque para a população mais vulnerável.

Tais fatos ressaltam que além de fornecer informações para a população sobre alimentação saudável, como é feito pelo Guia Alimentar para a População Brasileira ${ }^{8}$, é necessário implementar medidas públicas eficazes para promover melhor acesso a frutas e hortaliças variadas e de boa qualidade para os brasileiros, principalmente para os grupos populacionais mais vulneráveis socialmente. Uma tentativa inovadora neste sentido é a incorporação da variedade nos parâmetros nutricionais do $\mathrm{PNAE}^{9}$. Ações dessa natureza são de fundamental importância para que se diminuam as barreiras impostas para um consumo adequado e variado de frutas e hortaliças.

O presente estudo apresenta algumas limitações que devem ser mencionadas. Foram utilizados dados de aquisição, não de consumo de alimentos, o que não nos permite conhecer a distribuição intradomiciliar de consumo, que varia de acordo com faixa etária e sexo, e pode superestimar os valores encontrados, por não considerar o desperdício e o descarte de alimentos, por exemplo. Para minimizar este último problema, foram aplicados fatores de correção, a fim de excluir ao menos a fração não comestível dos alimentos. Outra limitação refere-se ao fato de que o consumo alimentar fora do domicílio não foi analisado, o que também influencia no valor final do consumo, subestimando os resultados. Porém, vale destacar que se trata de um estudo realizado com amostra representativa do Brasil e analisa não somente a quantidade absoluta - facilitando comparações com a recomendação da OMS - mas também a variedade de frutas e hortaliças adquiridas no país. A investigação desses dados permitiu também a identificação de grupos vulneráveis considerando as regiões e classes de rendimento. Ainda, o uso de dados de aquisição propiciou 
maior detalhamento sobre o consumo de frutas e hortaliças, considerando que em inquéritos de consumo individual alguns itens podem não aparecer por serem utilizados, principalmente, como parte de preparações, como cebola.

Conclui-se que a aquisição de frutas e hortaliças está abaixo do recomendado pela OMS nas cinco regiões brasileiras e em todas as classes de rendimento. Poucos itens representaram boa parte da aquisição desses alimentos, evidenciando pouca variedade na alimentação dos brasileiros.

Para melhorar essa situação, é necessário apropriar-se de meios que facilitem o acesso e permitam a diminuição do preço de frutas e hortaliças no Brasil. Além disso, deve ser incentivada a implementação de equipamentos públicos que aumentem a conveniência para a compra e que estimulem as hortas comunitárias. É necessário o investimento em ações educativas voltadas para o conhecimento sobre a ampla variedade de alimentos e suas propriedades, resgatando a mensagem da OMS de que o consumo adequado inclui ingestão regular, diversificada e quantitativamente recomendada de frutas e hortaliças, que estimulem o desenvolvimento de habilidades culinárias, por meio de preparações práticas e de baixo custo, e que divulguem informações de tecnologias que tornem a higienização, armazenamento, conservação, preparo e transporte de frutas e hortaliças mais práticos.

\section{Colaboradores}

Todas as autoras participaram substancialmente da elaboração do manuscrito. DS Canella: concepção e planejamento. N Oliveira, TR Paraizo e JP Sampaio: análise dos dados. N Oliveira, F Santin, TR Paraizo, JP Sampaio, N Moura-Nunes e DS Canella: interpretação dos resultados. N Oliveira e TR Paraizo: redação do artigo. N Oliveira, F Santin S, N Moura-Nunes e DS Canella': revisão crítica do artigo. N Oliveira, F Santin, TR Paraizo, JP Sampaio, N Moura-Nunes e DS Canella: aprovação da versão final encaminhada.

\section{Financiamento}

Coordenação de Aperfeiçoamento de Pessoal de Nível Superior - Brasil (CAPES) - Código de Financiamento 001. Fundação Carlos Chagas Filho de Amparo à Pesquisa do Estado do Rio de Janeiro (FAPERJ) - bolsa de doutorado de N Oliveira. Fundação de Amparo à Pesquisa do Estado de São Paulo (FAPESP) - bolsa de pós-doutorado de F Santin. Universidade do Estado do Rio de Janeiro (UERJ) - bolsa de iniciação científica de TR Paraizo e JP Sampaio. 


\section{Referências}

1. World Health Organization (WHO). Diet, nutrition and the prevention of chronic diseases. Geneva: WHO; 2003.

2. Brasil. Ministério da Saúde (MS). Secretaria de Vigilância em Saúde. Departamento de Análise de Situação de Saúde. Plano de ações estratégicas para o enfrentamento das doenças crônicas não transmissiveis (DCNT) no Brasil 2011-2022. Brasília: MS; 2011.

3. Miller V, Mente A, Dehghan M, Rangarajan S, Zhang X, Swaminathan S, Dagenais G, Gupta R, Mohan V, Lear S, Bangdiwala SI, Schutte AE, Wentzel-Viljoen E, Avezum A, Altuntas Y, Yusoff K, Ismail N, Peer N, Chifamba J, Diaz R, Rahman O, Mohammadifard N, Lana F, Zatonska K, Wielgosz A, Yusufali A, Iqbal R, Lopez-Jaramillo P, Khatib R, Rosengren A, Kutty VR, Li W, Liu J, Liu X, Yin L, Teo K, Anand S, Yusuf S, Prospective Urban Rural Epidemiology (PURE) study investigators. Fruit, vegetable, and legume intake, and cardiovascular disease and deaths in 18 countries (PURE): a prospective cohort study. Lancet 2017; 390(10107):2037-2049.

4. Vadiveloo M, Dixon LB, Mijanovich T, Elbel B, Parekh N. Dietary variety is inversely associated with body adiposity among US adults using a novel food diversity index. J Nutr 2015; 145(3):555-563.

5. Aune D, Giovannucci E, Boffetta P, Fadnes LT, Keum N, Norat T, Greenwood DC, Riboli E, Vatten LJ, Tonstad S. Fruit and vegetable intake and the risk of cardiovascular disease, total cancer and all-cause mortality-a systematic review and dose-response meta-analysis of prospective studies. Int J Epidemiol 2017; 46(3):1029-1056.

6. Rezende LF, Azeredo CM, Canella DS, Luiz OC, Levy RB, Eluf-Neto J. Coronary heart disease mortality, cardiovascular disease mortality and all-cause mortality attributable to dietary intake over 20 years in Brazil. Int J Cardiol 2016; 217:64-68.

7. Câmara Interministerial de Segurança Alimentar e Nutricional. Plano Nacional de Segurança Alimentar e Nutricional: 2016-2019. Brasília: CAISAN; 2017.

8. Brasil. Ministério da Saúde (MS). Secretaria de Atenção à Saúde. Departamento de Atenção Básica. Guia alimentar para a população brasileira. $2^{\mathrm{a}}$ ed. Brasília: MS; 2014.

9. Brasil. Fundo Nacional de Desenvolvimento da Educação. Resolução ${ }^{\circ}$ 6, de 08 de maio de 2020. Dispõe sobre o atendimento da alimentação escolar aos alunos da educação básica no âmbito do PNAE. Diário Oficial da União; 2020.

10. Levy RB, Claro RM, Mondini L, Sichieri R, Monteiro CA. Regional and socioeconomic distribution of household food availability in Brazil, in 2008-2009. Rev Saude Publica 2012; 46(1):6-15.

11. Canella DS, Louzada MLC, Claro RM, Costa JC, Bandoni DH, Levy RB, Martins APB. Consumption of vegetables and their relation with ultra-processed foods in Brazil. Rev Saude Publica 2018; 52:50.

12. Brasil. Ministério da Saúde (MS). Secretaria de Atenção à Saúde. Departamento de Atenção Básica. Alimentos regionais brasileiros. 2a ed. Brasília: MS; 2015.
13. Brasil. Ministério da Saúde (MS). Secretaria de Vigilância em Saúde. Departamento de Vigilância de Doenças e Agravos não Transmissíveis e Promoção da Saúde. Vigitel Brasil 2019: vigilância de fatores de risco e proteção para doenças crônicas por inquérito telefônico. Estimativas sobre frequência e distribuição sociodemográfica de fatores de risco e proteção para doenças crônicas nas capitais dos 26 estados brasileiros e no Distrito Federal em 2019. Brasília: MS; 2020.

14. Silva LES, Claro RM. Time trends in the consumption of fruits and vegetables among adults in Brazilian state capitals and the Federal District, 2008-2016. Cad Saude Publica 2019; 35(5):e00023618.

15. Claro RM, Monteiro CA. Family income, food prices, and household purchases of fruits and vegetables in Brazil. Rev Saude Publica 2010; 44(6):1014-1020.

16. Jaime PC, Stopa SR, Oliveira TP, Vieira ML, Szwarcwald CL, Malta DC. Prevalência e distribuição sociodemográfica de marcadores de alimentação saudável, Pesquisa Nacional de Saúde, Brasil 2013. Epidemiol Serv Saude 2015; 24(2):267-276.

17. Instituto Brasileiro de Geografia e Estatística (IBGE). Pesquisa de Orçamentos Familiares 2008-2009: Avaliação nutricional da disponibilidade domiciliar de alimentos no Brasil. Rio de Janeiro: IBGE; 2010.

18. Instituto Brasileiro de Geografia e Estatística (IBGE). Pesquisa de Orçamentos Familiares 2017-2018: Avaliação nutricional da disponibilidade domiciliar de alimentos no Brasil. Rio de Janeiro: IBGE; 2020.

19. Instituto Brasileiro de Geografia e Estatística (IBGE). Tabela de composição de alimentos - Estudo Nacional de Despesa Familiar. Rio de Janeiro: IBGE; 1996.

20. Koch W. Dietary polyphenols - important non-nutrients in the prevention of chronic noncommunicable diseases: a systematic review. Nutrients 2019; 11(5):1039.

21. VG, Tureck C, Locateli G, Peralta RM, Koehnlein EA. Estimate of consumption of phenolic compounds by Brazilian population. Rev Nutr 2015; 28(2):185-196.

22. Anacleto SL, Lajolo FM, Hassimotto NMA. Estimation of dietary flavonoid intake of the Brazilian population: a comparison between the USDA and Phenol-Explorer databases. J Food Compost Anal 2019; 78:1-8.

23. Chapman K, Goldsbury D, Watson W, Havill M, Wellard L, Hughes C, Bauman A, Allman-Farinelli $M$. Exploring perceptions and beliefs about the cost of fruit and vegetables and whether they are barriers to higher consumption. Appetite 2017; 113:310-319.

24. Brookie KL, Mainvil LA, Carr AC, Vissers MCM, Conner TS. The development and effectiveness of an ecological momentary intervention to increase daily fruit and vegetable consumption in low-consuming young adults. Appetite 2017; 108:32-41.

25. Lange D, Corbett J, Knoll N, Schwarzer R, Lippke S. Fruit and vegetable intake: the interplay of planning, social support, and sex. Int J Behav Med 2018; 25(4):421-430

26. Lee HA, Lim D, Oh K, Kim EJ, Park H. Mediating effects of metabolic factors on the association between fruit or vegetable intake and cardiovascular disease: the Korean National Health and Nutrition Examination Survey. BMJ Open 2018; 8(2):e019620. 
27. Santos GMGC, Silva AMR, Carvalho WO, Rech CR, Loch MR. Perceived barriers for the consumption of fruits and vegetables in Brazilian adults. Cien Saude Colet 2019; 24(7):2461-2470.

28. Figueira TR, Lopes ACS, Modena CM. Barreiras e fatores promotores do consumo de frutas e hortaliças entre usuários do Programa Academia da Saúde. Rev Nutr 2016; 29(1):85-95.

29. Mendonça RD, Lopes MS, Freitas PP, Campos SF, Menezes MC, Lopes ACS. Monotony in the consumption of fruits and vegetables and food environment characteristics. Rev Saude Publica. 2019; 53:63.

30. Food and Agriculture Organization of the United Nations (FAO). Influencing food environments for healthy diets. Roma: FAO; 2016.

31. Jaime PC, Duran AC, Sarti FM, Lock K. Investigating environmental determinants of diet, physical activity, and overweight among adults in Sao Paulo, Brazil. $J$ Urban Health 2011; 88(3):567-581.

32. United States Department of Agriculture (USDA). Ag and Food Statistics Charting the Essentials. Washington, D.C.: USDA; 2018.

Artigo apresentado em 15/07/2020

Aprovado em 23/09/2020

Versão final apresentada em 25/09/2020

Editores-chefes: Romeu Gomes, Antônio Augusto Moura da Silva 